\title{
CONTROLE DE Brevipalpus phoenicis (Geijskes, 1939) E Oligonychus ilicis (McGregor, 1917) (Acari: Tenuipalpidae, Tetranychidae) EM CAFEEIRO E O IMPACTO SOBRE ÁCAROS BENÉFICOS. II - SPIRODICLOFEN E AZOCYCLOTIN
}

\author{
Control of Brevipalpus phoenicis (Geijskes, 1939) and Oligonychus ilicis (McGregor, 1917) \\ (Acari: Tenuipalpidae, Tetranychidae) in coffee plants and the impact on beneficial mites. \\ II - Spirodiclofen and Azocyclotin
}

\author{
Paulo Rebelles Reis ${ }^{1}$, Marçal Pedro Neto ${ }^{2}$, Renato André Franco ${ }^{2}$
}

\begin{abstract}
RESUMO
O ácaro Brevipalpus phoenicis (Geijskes) é importante em cafeeiro (Coffea spp.) por ser o vetor do vírus da manchaanular, doença responsável por queda de folhas e má qualidade da bebida do café, e o ácaro-vermelho Oligonychus ilicis (McGregor) por reduzir a área foliar de fotossíntese. Ácaros da família Phytoseiidae, de várias espécies, são eficientes predadores associados aos ácaros-praga. Conduziu-se este trabalho com o objetivo de estudar o controle dos ácaros-praga com spirodiclofen e azocyclotin, e o impacto sobre fitoseídeos. Em laboratório foram estudados os efeitos ovicida, tópico, residual, tópico mais residual e a seletividade fisiológica aos fitoseídeos; em casa-de-vegetação foi avaliada a persistência no controle às duas espécies de ácaros-praga; e em campo foi avaliada a eficiência apenas no controle de B. phoenicis. Os bioensaios foram realizados em arenas de folhas destacadas. O efeito ovicida foi avaliado em ovos no início e final de incubação. Os efeitos residual, tópico e tópico mais residual foram avaliados pela mortalidade de larvas, ninfas e adultos aos oito dias, e a persistência até 30 dias após a aplicação. A seletividade aos fitoseídeos foi avaliada, pelo efeito na mortalidade e reprodução de fêmeas adultas, em teste residual em superfície de vidro. Spirodiclofen e azocyclotin (SC) mostraram eficiente ação ovicida, principalmente para ovos de B. phoenicis no início de incubação. Para ovos de O. ilicis, somente o spirodiclofen apresentou efeito ovicida. Em geral, os efeitos tópico e residual associados melhoraram a eficiência dos produtos no controle das fases pósembrionárias de ambas as espécies. O spirodiclofen apresentou seletividade aos ácaros predadores, já o azocyclotin foi nocivo. Em campo, ambos os acaricidas mostram-se altamente eficientes na redução de todas as fases pós-embrionárias do ácaro $B$. phoenicis, principalmente nas folhas.
\end{abstract}

Termos para indexação: Ácaro, mancha-anular, controle químico, seletividade, Coffea arabica.

\begin{abstract}
The mite Brevipalpus phoenicis (Geijskes) is important in coffee plants (Coffea spp.) for being the vector of the coffee ringspot virus, disease responsible for leaf fall and bad quality of the coffee beverage. The red spider mite Oligonychus ilicis (McGregor) is also important for reducing the area of photosynthesis in leaves. Mites of the family Phytoseiidae are efficient predators associated to the pest-mites. The objective of this work was to study the control of the pest-mites with spirodiclofen and azocyclotin, and the impact on phytoseiids. Studies were carried out in laboratory conditions on the ovicidal, topical, residual, topical plus residual effects, and the physiological selectivity for the phytoseiids. The persistence of the products in the control of the pest-mites was studied in semi-field conditions. In field-test only was evaluated the efficiency in the control of $B$. phoenicis. Bioassays were made in detached leaves. The ovicidal effect was evaluated in eggs in the beginning and end of incubation; the residual, topical and topical plus residual effects on larvae, nymphs and adult were assessed through mortality evaluation eight days after spraying, while the persistence was evaluated until 30 days after spraying. The phytoseiids selectivity was evaluated in a glass surface residual test through the adult female's mortality and reproduction effect. Spirodiclofen and azocyclotin (SC) showed efficient ovicidal action, mainly for eggs of B. phoenicis in the beginning of incubation. For eggs of $O$. ilicis only the spirodiclofen presented ovicidal effect. In general, the associated topical plus residual effects improve the performance of the products in the control of the post-embryonic phases of both species. The spirodiclofen presented selectivity for the predator mites; the azocyclotin was already noxious. In the field, both acaricides showed highly efficiency in the reduction of all the post-embryonic phases of the pest-mite B. phoenicis, mainly in the leaves.
\end{abstract}

Index terms: Mite, coffee ringspot virus, chemical control, selectivity, Coffea arabica.

(Recebido para publicação em $1^{\circ}$ de abril de 2004 e aprovado em 23 de fevereiro de 2005)

\footnotetext{
1. Engenheiro Agrônomo, D.Sc., Pesquisador EPAMIG-CTSM/EcoCentro - bolsista do CNPq - Caixa Postal 176 - $37200-000$ - Lavras, MG paulo.rebelles@epamig.ufla.br

2. Bolsistas de IC / CBP\&D-Café - Estudantes de Agronomia da Universidade Federal de Lavras/UFLA - Caixa Postal 3037 - $37200-000$ Lavras, MG.
} 


\section{INTRODUÇÃO}

O ácaro Brevipalpus phoenicis (Geijskes, 1939) (Acari: Tenuipalpidae) tem sido relatado vivendo em cafeeiros (Coffea spp.) no Brasil desde 1950 (AMARAL, 1951), quando foi relatado no Estado de São Paulo juntamente com surtos do ácaro-vermelho Oligonychus ilicis (McGregor, 1917) (Acari: Tetranychidae). Posteriormente, a associação do ácaro B. phoenicis à doença mancha-anular do cafeeiro foi estudada por Chagas (1973).

Desde 1990, com destaque para 1995, a infestação de B. phoenicis e da mancha-anular têm sido relatadas em Minas Gerais, causando intensa desfolha em cafeeiros, principalmente na região do Alto Paranaiba (FIGUEIRA et al., 1996), sendo também constatada a presença do ácaro nas demais regiões cafeeiras do Brasil, tanto em cafeeiro Arábica (Coffea arabica L.) quanto em Canéfora (Coffea canephora Pierre \& Froenher) (MATIELLO, 1987).

B. phoenicis é importante em cafeeiro por ser o vetor do vírus da mancha-anular, doença responsável por queda de folhas e má qualidade da bebida do café (REIS \& CHAGAS, 2001).

O ácaro-vermelho já foi referido como a segunda praga em importância para o cafeeiro Conillon ( $C$. canephora) no Estado do Espirito Santo (IBC, 1985); esse cafeeiro é considerado mais sensível ao ácaro do que o Arábica (C. arabica). Períodos de seca, com estiagem prolongada, são condições propícias à proliferação de $O$. ilicis, podendo causar desfolha das plantas, sendo que lavouras novas, em formação, terão seu desenvolvimento retardado (REIS \& SOUZA, 1986).

Ácaros da família Phytoseiidae estão associados a ácaros do cafeeiro (PALLINI FILHO et al., 1992; REIS et al., 2000a) e são os mais importantes e estudados predadores de ácaros-praga (McMURTRY \& CROFT, 1997).

O controle químico de ácaros em cafeeiros no Brasil, principalmente de $B$. phoenicis, é muito pouco conhecido, ao contrário do que ocorre em citros. Alguns acaricidas como cyhexatin, azocyclotin, bromopropilato, fenpyroximate e meothrin mostraram ser eficientes no controle de B. phoenicis em cafeeiro (OLIVEIRA \& REIFF, 1998; PAPA, 1997), porém não foi relatada a seletividade aos predadores. Reis et al. (2002) destacaram como eficientes no controle de $B$. phoenicis, e seletivos aos fitoseídeos, o hexythiazox, fenbutatin oxide, enxofre e abamectin.

Considerando os fatos expostos, objetivou-se com este estudo avaliar o efeito dos acaricidas spirodiclofen (Envidor 240 SC) e azocyclotin (Caligur 500 SC) sobre os ácaros-praga do cafeeiro, B. phoenicis e $O$. ilicis, e sua seletividade a ácaros predadores pertencentes à família Phytoseiidae, Euseius alatus DeLeon, 1966, Euseius citrifolius Denmark \& Muma, 1970 e Amblyseius herbicolus (Chant, 1959) a eles associados.

\section{MATERIAL E MÉTODOS}

Os experimentos foram realizados no Centro de Pesquisa em Manejo Ecológico de Pragas e Doenças de Plantas - EcoCentro, da Empresa de Pesquisa Agropecuária de Minas Gerais - EPAMIG, no Campus da UFLA. À exceção do estudo da persistência em casade-vegetação e da eficiência em campo, os demais experimentos foram feitos em laboratório a $25 \pm 2{ }^{\circ} \mathrm{C}$, $70 \pm 10 \%$ de UR e 14 horas de fotofase.

Criação de manutenção: A criação de manutenção do ácaro-praga B. phoenicis, para uso nos bioensaios, foi feita em laranjas parafinadas, conforme metodologia descrita por Chiavegato (1986). A criação de $O$. ilicis foi feita em folhas de cafeeiro, conforme metodologia descrita por Reis et al. (1997). Os ácaros predadores pertencentes à família Phytoseiidae, utilizados nos testes, foram oriundos de criação de manutenção em laboratório, realizada conforme metodologia descrita por Reis \& Alves (1997).

Procedimentos Experimentais no Laboratório: Os testes de efeito ovicida, tópico, residual e tópico mais aplicados aos ácaros-praga, foram realizados em folhas de cafeeiro (C. arabica), coletadas em plantas sem tratamento fitossanitário. As folhas serviram de arena e fonte de alimento aos ácaros, e foram colocadas sobre uma esponja de $2 \mathrm{~cm}$ de espessura, constantemente umedecida em água destilada, que ocupava todo o interior de uma placa de Petri de $15 \mathrm{~cm}$ de diâmetro por 2 $\mathrm{cm}$ de profundidade, sem tampa, conforme metodologia descrita por Reis et al. (1997). Os produtos estudados, e dosagens por hectare, encontram-se relatados na Tabela 1. A aplicação foi feita em torre de Potter a uma pressão de $15 \mathrm{lb} / \mathrm{pol}^{2}$, com a mesa de pulverização a uma distância de 1,7 cm do tubo de pulverização. Exceto no estudo do efeito tópico, no qual somente os ácaros foram pulverizados, cada folha recebeu um depósito de calda da ordem de 1,68 $\pm 0,36 \mathrm{mg} / \mathrm{cm}^{2}$, em conformidade com o proposto pela IOBC/WPRS (HASSAN et al., 1994), representando o que ocorre quando é feita uma aplicação em campo. Para cada tratamento foram utilizadas seis repetições. 
Efeito ovicida: Fêmeas adultas dos ácaros foram confinadas nas arenas durante dois dias para a obtenção de ovos, dos quais foram aproveitados 20 por arena. Os ovos assim obtidos foram pulverizados com os produtos em teste e observados diariamente até a eclosão de larvas. Foram feitos testes com ovos de $B$. phoenicis com dois e com oito dias de idade, ou seja, ovos no início e no final do período embrionário (CHIAVEGATO, 1986) e com ovos de O. ilicis com dois e seis dias de idade, também início e fim do período embrionário (REIS et al., 1997). Quando houve eclosão de larva, o produto foi considerado sem efeito ovicida.

Efeito tópico: Foi estudado o efeito dos produtos quando aplicados diretamente sobre o idiossoma do ácaro. Após a pulverização, 10 ácaros de cada fase pós-embrionária (larva, ninfa e adulto) foram colocados em arenas não pulverizadas, em experimentos independentes para cada fase e espécie. Somente após oito dias da aplicação foi avaliada a mortalidade, devido ao início de ação do spirodiclofen ser mais retardado que dos acaricidas de efeito agudo (WACHENDORFF et al., 2002).

Efeito residual: Neste experimento foi estudado o efeito residual dos produtos sobre as folhas na mortalidade do ácaro. Depois da pulverização as folhas foram secas em condições ambientes do laboratório por 1 hora. Foram colocados 10 ácaros de cada fase pósembrionária, em cada uma das três partes em que foi dividida cada folha, em experimentos independentes para cada fase e espécie, avaliando-se a mortalidade oito dias mais tarde.

Efeito tópico mais residual: Neste experimento foram estudados os dois efeitos em conjunto. Dez ácaros de cada fase pós-embrionária, em experimentos independentes para cada fase e espécie, foram colocadas nas arenas antes da pulverização. Os ácaros receberam o produto sobre o idiossoma e permaneceram em contato com o resíduo do mesmo sobre a folha, avaliando-se a mortalidade oito dias mais tarde.

Persistência: Em casa-de-vegetação foi avaliada a persistência de cada produto no controle dos ácaros da mancha-anular e vermelho do cafeeiro. Foram utilizadas mudas de cafeeiro (C. arabica) de aproximadamente $100 \mathrm{~cm}$ de altura, plantadas em vasos plásticos de 10 litros, em casa-de-vegetação. Estas foram pulverizadas uma única vez com os mesmos produtos e dosagens utilizadas nos bioensaios em laboratório (Tabela 1). Os produtos foram aplicados até o ponto de escorrimento, utilizando-se um pulverizador de pressão constante com capacidade de um litro. Após a aplicação de cada produto, o pulverizador foi lavado com água corrente, e depois com álcool absoluto, a fim de se evitar o efeito de resíduos do produto utilizado sobre outros produtos a serem aplicados posteriormente.

Os bioensaios realizados para verificar a persistência, dos acaricidas foram feitos aos $0,5,15$ e 30 dias após a aplicação (DAA) em folhas destacadas das plantas, sendo os vasos mantidos em casa-devegetação. Em laboratório, das folhas destacadas, foram confeccionadas arenas de $3 \mathrm{~cm}$ de diâmetro. As arenas foram colocadas a flutuar em água em uma placa de Petri de $15 \mathrm{~cm}$ de diâmetro x $2 \mathrm{~cm}$ de profundidade, sem tampa, conforme metodologia descrita por Reis et al. (1998). Para cada tratamento foram utilizadas seis repetições. Em cada arena foram colocados 10 ácaros de cada fase a ser avaliada (larva, ninfa e adulta), oriundos da criação de manutenção, em experimentos independentes para cada espécie. Após oito dias foram contados os ácaros sobreviventes.

Após os testes, cada produto foi classificado em uma das quatro classes de persistência (HASSAN et al., 1994), de acordo com o período em que pelo menos 80\% dos ácaros morreram: classe 1 - baixa persistência, (até 5 dias após a aplicação); classe 2 - levemente persistente (entre 6 e 15 dias); classe 3 - moderadamente persistente (entre 16 e 30 dias) e classe 4 - persistentes, para produtos que alcançaram um eficiente controle dos ácaros além de 30 DAA.

Seletividade a ácaros predadores: Foi estudada a seletividade sobre três espécies de ácaros predadores pertencentes à família Phytoseiidae comumente associadas aos ácaros-praga do cafeeiro, E. alatus, E. citrifolius e $A$. herbicolus. Os ácaros fitoseídeos utilizados nos testes foram oriundos de criação de manutenção em laboratório. Foi utilizado o método residual da pulverização em superfície de vidro, recomendado como padrão para testes em laboratório de efeitos adversos de produtos fitossanitários a ácaros predadores, pela IOBC / WPRS (HASSAN et al., 1994). Lamínulas de vidro de 20x20 mm, flutuando em água em placa de Petri de $5 \mathrm{~cm}$ de diâmetro x $2 \mathrm{~cm}$ de profundidade, sem tampa, foram usadas como superfície para aplicação dos produtos, e suporte para os ácaros (REIS et al., 1998, 1999). O efeito residual foi medido por meio do efeito adverso ou total (E\%), calculado levando em conta a mortalidade no tratamento e o efeito na reprodução (OVERMEER \& ZON, 1982). Durante oito dias, foram diariamente contadas as fêmeas vivas, e retiradas as mortas, bem como o número de ovos 
viáveis (que deram origem a larvas). Os valores dos efeitos totais, encontrados para cada produto testado, foram classificados nas classes de 1 a 4, conforme critérios estabelecidos pela IOBC / WPRS para enquadrar produtos fitossanitários quanto ao efeito adverso causado a organismos benéficos em testes de laboratório (HASSAN et al., 1994), sendo: classe $1=\mathrm{E}$ $<30 \%$ (inócuo ou não nocivo); classe $2=30 \%<\mathrm{E}<$ 79\% (levemente nocivo); classe $3=80 \%<\mathrm{E}<99 \%$ (moderadamente nocivo) e classe $4=\mathrm{E}>99 \%$ (nocivo).

Campo: O efeito dos produtos em campo foi avaliado apenas para $B$. phoenicis, em experimento instalado em cafezal 'Catuaí' em produção, localizado no município de Ijaci, região Sul de Minas Gerais. O delineamento experimental foi o de blocos ao acaso, com cinco repetições. Entre um bloco e outro foi deixada uma linha de cafeeiros como bordadura. Cada parcela constou de sete plantas, sendo úteis as cinco centrais. Os tratamentos foram aplicados uma só vez, com atomizador costal motorizado e gasto de 1000 litros de calda por hectare, pois é necessário alto volume de calda acaricida para melhor eficiência no controle do ácaro (OLIVEIRA \& REIFF, 1998). O efeito de cada produto no controle do ácaro $B$. phoenicis foi avaliado por meio de contagem, em laboratório sob microscópio estereoscópico, de ácaros e ovos em 25 folhas e cinco ramos por parcela, coletados no terço inferior das plantas, local do cafeeiro onde ocorre a maior quantidade de ácaros dessa espécie (REIS et al., 2000b). Para efeito de análise estatística os dados foram transformados em $\sqrt{x+0,5}$ e as médias comparadas pelo teste de Duncan $(\mathrm{P}<0,05)$. A porcentagem de eficiência foi calculada conforme Abbott (1925).

\section{RESULTADOS E DISCUSSÃO}

Efeito ovicida: Spirodiclofen (Envidor 240 SC) e azocyclotin (Caligur 500 SC) apresentaram excelente efeito ovicida sobre $B$. phoenicis, com eficiência de controle entre 91 e 100\%, tanto para ovos no início ou final do período embrionário. Azocyclotin (Peropal 250 PM) apresentou maior eficiência ovicida sobre ovos no início do período embrionário (87\% de controle), assim como o hexythiazox (Savey 500 PM) considerado padrão ovicida neste experimento, com $100 \%$ de controle. A formulação SC do azocyclotin apresentou melhor desempenho como ovicida que a $\mathrm{PM}$, e como a dosagem em princípio ativo foi a mesma para as duas formulações a diferença encontrada pode ser devida a uma melhor aderência e penetração nos ovos da formulação SC. O fenbutatin oxide (Torque 500 SC) apresentou cerca de 70\% de eficiência como ovicida, para ovos com dois ou oito dias de período embrionário (Tabela 1). O ótimo efeito ovicida do hexythiazox e parcial do fenbutatin oxide para $B$. phoenicis já foi relatado também por Reis et al. (2002).

Para O. ilicis somente o spirodiclofen apresentou efeito ovicida, com $100 \%$ de controle, porém apenas para ovos no início do período embrionário (Tabela 2), o que demonstra maior resistência de ovos no final do período embrionário e maior resistência de ovos de O. ilicis aos acaricidas que ovos de B. phoenicis.

TABELA 1 - Número médio de larvas de Brevipalpus phoenicis eclodidas de ovos pulverizados, após dois e oito dias da oviposição e eficiência ovicida (\% Efic.) de spirodiclofen, azocyclotin, fenbutatin oxide e hexythiazox (n = 20 ovos).

\begin{tabular}{|c|c|c|c|c|c|}
\hline \multirow{2}{*}{ Tratamentos } & \multirow{2}{*}{$\begin{array}{c}\text { Dosagem } \\
\text { por ha }^{1}\end{array}$} & \multicolumn{2}{|c|}{ Dois dias } & \multicolumn{2}{|c|}{ Oito dias } \\
\hline & & Média $^{2}$ & \% Efic. & Média $^{2}$ & \% Efic. \\
\hline Testemunha & - & $19,3 \mathrm{a}$ & - & $18,8 \mathrm{a}$ & - \\
\hline Spirodiclofen (Envidor 240 SC) & 300 & $0,0 \quad \mathrm{~d}$ & 100,0 & $0,3 \mathrm{c}$ & 98,4 \\
\hline Azocyclotin (Peropal 250 PM) ${ }^{3}$ & 1000 & $2,2 \mathrm{c}$ & 88,6 & $8,2 \mathrm{~b}$ & 56,4 \\
\hline Azocyclotin (Caligur 500 SC) & 500 & $0,5 \mathrm{~d}$ & 97,4 & $1,7 \mathrm{c}$ & 91,0 \\
\hline Fenbutatin oxide (Torque $500 \mathrm{SC})^{3}$ & 800 & $5,2 \mathrm{~b}$ & 73,1 & $5,3 \mathrm{~b}$ & 71,8 \\
\hline Hexythiazox (Savey 500 PM) ${ }^{3}$ & 30 & $0,0 \mathrm{~d}$ & 100,0 & $5,5 \mathrm{~b}$ & 70,7 \\
\hline CV (\%) & & 22,2 & & 21,1 & \\
\hline
\end{tabular}

${ }^{1}$ g ou mL do produto comercial por 1000 litros de água.

${ }^{2}$ Médias seguidas de mesma letra na coluna, não diferem entre si, pelo teste de Duncan $(\mathrm{P}<0,05)$.

${ }^{3}$ Padrões de controle neste experimento. 
TABELA 2 - Número médio de larvas de Oligonychus ilicis eclodidas de ovos pulverizados após dois e seis dias da oviposição e eficiência ovicida (\% Efic.) de spirodiclofen, azocyclotin, fenbutatin oxide e hexythiazox ( $\mathrm{n}=20$ ovos).

\begin{tabular}{|c|c|c|c|c|c|}
\hline \multirow{2}{*}{ Tratamentos } & \multirow{2}{*}{$\begin{array}{c}\text { Dosagem } \\
\text { por ha }^{1}\end{array}$} & \multicolumn{2}{|c|}{ Dois dias } & \multicolumn{2}{|c|}{ Seis dias } \\
\hline & & Média $^{2}$ & \% Efic. & Média $^{2}$ & \% Efic. \\
\hline Testemunha & - & 18,2 a & - & 19,2 a & - \\
\hline Spirodiclofen (Envidor 240 SC) & 300 & $0,0 \mathrm{c}$ & 100,0 & $15,3 \mathrm{ab}$ & 20,3 \\
\hline Azocyclotin (Peropal 250 PM) ${ }^{3}$ & 1000 & 17,5 a & 3,9 & $13,5 \mathrm{~b}$ & 29,7 \\
\hline Azocyclotin (Caligur 500 SC) & 500 & $11,0 \mathrm{~b}$ & 39,6 & $7,7 \quad \mathrm{c}$ & 59,9 \\
\hline Fenbutatin oxide (Torque 500 SC) ${ }^{3}$ & 800 & $13,2 \mathrm{ab}$ & 27,5 & $14,7 \mathrm{ab}$ & 23,4 \\
\hline Hexythiazox (Savey $500 \mathrm{PM})^{3}$ & 30 & $17,0 \mathrm{a}$ & 6,6 & $17,0 \mathrm{ab}$ & 11,5 \\
\hline CV (\%) & & 22,4 & & 15,3 & \\
\hline
\end{tabular}

${ }^{1} \mathrm{~g}$ ou $\mathrm{mL}$ do produto comercial por 1000 litros de água.

${ }^{2}$ Médias seguidas de mesma letra na coluna, não diferem entre si, pelo teste de Duncan $(\mathrm{P}<0,05)$.

${ }^{3}$ Padrões de controle neste experimento.

Efeito tópico: O hexythiazox não apresentou efeito de controle quando aplicado sobre as fases pósembrionárias de $B$. phoenicis (12 a 25\%) e de O. ilicis (2,4 a 12\%). O azocyclotin (PM e SC) e fenbutatin oxide apresentaram alto efeito sobre $B$. phoenicis, com $100 \%$ de eficiência para o controle de larvas, ninfas e adultos e $100 \%$ de mortalidade para larvas e ninfas de $O$. ilicis. O spirodiclofen apresentou eficiência sobre larvas de B. phoenicis (96\%) e de O. ilicis (81\%), mas não para ninfas (43 a 52\%) e adultos (19 a 22\%).

Efeito residual: $\mathrm{O}$ efeito residual dos produtos sobre as fases imaturas de $B$. phoenicis e $O$. ilicis foi semelhante àquele obtido com o efeito tópico, ou seja, azocyclotin (PM e SC) e fenbutatin oxide com $100 \%$ de eficiência no controle de todas as fases pósembrionárias, e baixa eficiência do hexythiazox (3,6 a $39 \%)$. Spirodiclofen foi mais eficiente no controle de larvas e ninfas (85 a 100\%), mas não apresentou controle de adultos das duas espécies de ácaros (16 a $47 \%)$.

Efeito tópico mais residual: Em geral, o efeito tópico associado ao efeito residual melhorou a eficiência dos tratamentos no controle das fases pósembrionárias de $B$. phoenicis e O. ilicis (80 a 100\%), à exceção do spirodiclofen para o controle de adultos (12 a 58\%). O menor efeito de mortalidade de adultos pelo spirodiclofen talvez seja devido ao modo de ação do produto, que tem demonstrado que não é neurotóxico e atua inibindo a biosíntese de lípídeos (WACHENDORFF et al., 2002). Esse modo de ação talvez explique porque os ácaros imaturos não passaram de um estádio do desenvolvimento para outro, fato observado neste trabalho, e houve menor mortalidade de adultos, ou seja, atua no desenvolvimento do ácaro. O hexythiazox não apresentou controle das fases pósembrionárias de ambas as espécies de ácaros (17 a 33\%), efeito também já relatado por Reis et al. (2002) para B. phoenicis.

Persistência: $O$ azocyclotin nas duas formulações testadas (PM e SC) e fenbutatin oxide apresentaram-se persistentes no controle de todas as fases pós-embrionárias de B. phoenicis e O. ilicis (eficiência > 80\% além de 30 DAA). O spirodiclofen foi persistente para larvas e ninfas de $B$. phoenicis e O. ilicis, moderadamente persistente no controle de adultos de $B$. phoenicis (16 a 30 dias) e não mostrou eficiência no controle de adultos de $O$. ilicis, sendo este resultado semelhante aos obtidos nos testes de efeitos residual, tópico e tópico mais residual. O hexythiazox não exerceu controle das fases pós-embrionárias de ambas as espécies de ácaros. Embora a mortalidade de fêmeas adultas de $O$. ilicis causada pelo spirodiclofen tenha sido baixa (24\%), foi observada uma redução acentuada no número e na viabilidade dos ovos. Wachendorff et al. (2002) reportaram que concentrações subletais de spirodiclofen 
nas fêmeas influem na fecundidade, de modo que não ovipositam ou só depositam ovos estéreis. A explicação para a redução da fecundidade e esterilidade de ovos pode ser devida ao modo de ação já relatado anteriormente para os efeitos tópico e residual, ou seja, o produto influi na biosíntese de lipídeos impedindo o desenvolvimento de imaturos e nos adultos influi no processo reprodutivo. Por esta razão, não se deve esperar efeito imediato na redução da população de ácaros com o uso de spirodiclofen.

Seletividade: $O$ acaricida spirodiclofen foi levemente nocivo (classe 2) às espécies $E$. citrifolius e A. herbicolus, igualando-se ao fenbutatin oxide (padrão de seletividade neste trabalho), e para E. alatus foi moderadamente nocivo (classe 3 ). $\mathrm{O}$ hexythiazox mostrou-se inócuo (classe 1) para E. citrifolius e E. alatus, e levemente nocivo (classe 2) para $A$. herbicolus. Apenas o azocyclotin (PM e SC) apresentou-se nocivo às três espécies de ácaros predadores estudadas. A seletividade do fenbutatin oxide e do hexythiazox aos fitoseídeos também já foi constatada por Reis et al. (1998, 1999). Apesar da baixa mortalidade de fêmeas adultas de fitoseídeos, o tratamento com spirodiclofen reduziu o número de ovos postos em relação ao hexythiazox e fenbutatin oxide (Tabela 3, 4 e 5), provavelmente pelo mesmo efeito relatado para os ácaros-praga, porém, com menos intensidade. Embora o spirodiclofen não tenha sido inócuo aos fitoseídeos, Wolf \& Schnorbach (2002) consideram que o produto não representa risco para esses predadores, pois os efeitos sobre eles são baixos e as populações afetadas se recuperam rapidamente.
Campo: À exceção do hexythiazox, já aos 5 DAA (dias após a aplicação) todos os demais produtos testados mostraram efeito de controle acima de $80 \%$, nas folhas do cafeeiro, para todas as fases pósembrionárias de B. phoenicis. Essa eficiência continuou e até aumentou aos 15 DAA. Nos ramos, aos 5 DAA, como já era esperado, pelo efeito de abrigo que proporciona aos ácaros, as eficiências foram menores, porém muito próximas de $80 \%$, considerada como ideal neste trabalho. Aos 15 DAA, somente o spirodiclofen continuou com alta eficiência de controle dos ácaros nos ramos (Tabela 6). A eficiência do spirodiclofen no controle de $O$. ilicis em condições de campo já foi relatada por Matiello (2000), constatando boa eficiência, porém, lenta devido ao tipo de efeito que o produto possui, como já discutido anteriormente. A eficiência no controle das fases pós-embrionárias de $B$. phoenicis nas folhas teve como conseqüência a redução do número de ovos, ressaltando a eficiência dos produtos no controle do ácaro. Nos ramos, a exemplo do que ocorreu no controle das fases pósembrionárias, a redução do número de ovos foi menor, devido o ácaro ficar menos exposto aos produtos, encontrando abrigo nas fendas dos ramos. Mesmo assim, aos 15 DAA a redução do número de ovos foi além de $80 \%$, com exceção do hexythiazox, por este produto não exercer controle das fases pósembrionárias (Tabela 7). A redução do número de ovos expressa a eficiência na mortalidade de fases pós-embrionárias, principalmente adultos, e como conseqüência haverá redução no número de ovos depositados.

TABELA 3 - Toxicidade de spirodiclofen, azocyclotin, fenbutatin oxide e hexythiazox ao predador Euseius citrifolius em teste residual de laboratório a $25 \pm 2^{\circ}$ C, $70 \pm 10 \%$ de UR e 14 horas de fotofase (resíduo de 1,68 $\pm 0,36$ $\mathrm{mg} / \mathrm{cm}^{2}$ em superfície de vidro).

\begin{tabular}{lcccccc}
\hline \multicolumn{1}{c}{ Nome técnico } & $\begin{array}{c}\text { Dosagem } \\
\mathbf{p o r ~ h a}^{\mathbf{1}}\end{array}$ & $\begin{array}{c}\mathbf{M}_{\mathbf{c}} \mathbf{c}^{\mathbf{2}} \\
\mathbf{( \% )}\end{array}$ & $\mathbf{R}^{\mathbf{3}}$ & $\mathbf{E}_{\mathbf{r}}{ }^{\mathbf{4}}$ & $\begin{array}{c}\mathbf{E}^{\mathbf{5}} \\
\mathbf{( \% )}\end{array}$ & Classe $^{\mathbf{6}}$ \\
\hline Hexythiazox (Savey 500 PM) & 30 & 0,00 & 6,70 & 0,84 & 15,90 & 1 \\
Fenbutatin oxide (Torque 500 SC) & 800 & 20,00 & 5,70 & 0,72 & 42,68 & 2 \\
Spirodiclofen (Envidor 240 SC) & 300 & 3,33 & 4,14 & 0,52 & 49,37 & 2 \\
Azocyclotin (Peropal 250 PM) & 1000 & 100,00 & 0,00 & 0,00 & 100,00 & 4 \\
Azocyclotin (Caligur 500 SC) & 500 & 100,00 & 0,00 & 0,00 & 100,00 & 4 \\
\hline
\end{tabular}

1g ou $\mathrm{mL}$ do produto comercial/1000 litros de água.

${ }^{2} \mathrm{M}_{\mathrm{c}}=$ Mortalidade corrigida (\%). $\mathrm{M}_{\mathrm{c}}=$ (ácaros vivos test. - ácaros vivos trat.)/ácaros vivos test. $\mathrm{x} 100$.

${ }^{3} \mathrm{R}=$ Produção média de ovos/fêmeas. $\mathrm{R}=\mathrm{n}^{0}$ ovos viáveis/ $\mathrm{n}^{\mathrm{o}}$ de fêmeas.

${ }^{4}$ Efeito na reprodução. $\mathrm{Er}=\mathrm{R}_{\text {Tratamento }} / \mathrm{R}_{\text {Testemunha }}$

${ }^{5}$ Efeito total ou adverso. $\mathrm{E}=100 \%-(100 \%-\mathrm{Mc}) \times \mathrm{Er}$.

${ }^{6}$ Classes toxicidade segundo a IOBC/WPRS: Classe $1=\mathrm{E}<30 \%$ (não nocivo); Classe $2=30<\mathrm{E}<80$ (levemente nocivo); Classe $3=80<\mathrm{E}<99$ (moderadamente nocivo); Classe 4 = E $>99 \%$ (nocivo). 
REIS, P. R. et al.

TABELA 4 - Toxicidade de spirodiclofen, azocyclotin, fenbutatin oxide e hexythiazox ao predador Euseius alatus em teste residual de laboratório a $25 \pm 2^{\circ} \mathrm{C}, 70 \pm 10 \%$ de UR e 14 horas de fotofase (resíduo de 1,68 $\pm 0,36 \mathrm{mg} / \mathrm{cm}{ }^{2} \mathrm{em}$ superfície de vidro).

\begin{tabular}{lcccccc}
\hline \multicolumn{1}{c}{ Nome técnico } & $\begin{array}{c}\text { Dosagem } \\
\mathbf{p o r ~ h a}^{\mathbf{1}}\end{array}$ & $\begin{array}{c}\mathbf{M}_{\mathbf{c}}{ }^{2} \\
\mathbf{( \% )}\end{array}$ & $\mathbf{R}^{\mathbf{3}}$ & $\mathbf{E}_{\mathbf{r}}{ }^{4}$ & $\begin{array}{c}\mathbf{E}^{\mathbf{5}} \\
\mathbf{( \% )}\end{array}$ & Classe $^{\mathbf{6}}$ \\
\hline Hexythiazox (Savey 500 PM) & 30 & 0,00 & 5,36 & 0,76 & 23,86 & 1 \\
Fenbutatin oxide (Torque 500 SC) & 800 & 85,71 & 10,00 & 1,42 & 79,70 & 2 \\
Spirodiclofen (Envidor 240 SC) & 300 & 17,86 & 1,21 & 0,17 & 85,79 & 3 \\
Azocyclotin (Peropal 250 PM) & 1000 & 100,00 & 0,00 & 0,00 & 100,00 & 4 \\
Azocyclotin (Caligur 500 SC) & 500 & 100,00 & 0,00 & 0,00 & 100,00 & 4 \\
\hline
\end{tabular}

${ }^{1} \mathrm{~g}$ ou mL do produto comercial/1000 litros de água.

${ }^{2} \mathrm{M}_{\mathrm{c}}=$ Mortalidade corrigida (\%). $\mathrm{M}_{\mathrm{c}}=$ (ácaros vivos test. - ácaros vivos trat.)/ácaros vivos test. $\mathrm{x} 100$.

${ }^{3} \mathrm{R}=$ Produção média de ovos/fêmeas. $\mathrm{R}=\mathrm{n}^{0}$ ovos viáveis/ $\mathrm{n}^{\underline{0}}$ de fêmeas.

${ }^{4}$ Efeito na reprodução. $\mathrm{Er}=\mathrm{R}_{\text {Tratamento }} / \mathrm{R}_{\text {Testemunha }}$

${ }^{5}$ Efeito total ou adverso. $\mathrm{E}=100 \%$ - $(100 \%$ - Mc) $\mathrm{x}$ Er.

${ }^{6}$ Classes toxicidade segundo a IOBC/WPRS: Classe $1=\mathrm{E}<30 \%$ (não nocivo); Classe $2=30<\mathrm{E}<80$ (levemente nocivo); Classe $3=80<\mathrm{E}<99$ (moderadamente nocivo); Classe $4=\mathrm{E}>99 \%$ (nocivo).

TABELA 5 - Toxicidade de spirodiclofen, azocyclotin, fenbutatin oxide e hexythiazox ao predador Amblyseius herbicolus em teste residual de laboratório a $25 \pm 2^{\circ} \mathrm{C}, 70 \pm 10 \%$ de UR e 14 horas de fotofase (resíduo de 1,68 $\pm 0,36 \mathrm{mg} /$ $\mathrm{cm}^{2}$ em superfície de vidro).

\begin{tabular}{lcccccc}
\hline \multicolumn{1}{c}{ Nome técnico } & $\begin{array}{c}\text { Dosagem } \\
\text { por ha }\end{array}$ & $\begin{array}{c}\mathbf{M}_{\mathbf{c}}{ }^{\mathbf{2}} \\
\mathbf{( \% )}\end{array}$ & $\mathbf{R}^{\mathbf{3}}$ & $\mathbf{E}_{\mathbf{r}}{ }^{4}$ & $\begin{array}{c}\mathbf{E}^{\mathbf{5}} \\
\mathbf{( \% )}\end{array}$ & Classe $^{\mathbf{6}^{6}}$ \\
\hline Hexythiazox (Savey 500 PM) & 30 & 28,00 & 4,06 & 0,82 & 40,65 & 2 \\
Fenbutatin oxide (Torque 500 SC) & 800 & 68,00 & 6,50 & 1,32 & 57,72 & 2 \\
Spirodiclofen (Envidor 240 SC) & 300 & 44,00 & 2,57 & 0,52 & 70,73 & 2 \\
Azocyclotin (Peropal 250 PM) & 1000 & 100,00 & 0,00 & 0,00 & 100,00 & 4 \\
Azocyclotin (Caligur 500 SC) & 500 & 100,00 & 0,00 & 0,00 & 100,00 & 4 \\
\hline
\end{tabular}

$1 \mathrm{~g}$ ou $\mathrm{mL}$ do produto comercial/1000 litros de água.

${ }^{2} \mathrm{M}_{\mathrm{C}}=$ Mortalidade corrigida (\%). $\mathrm{M}_{\mathrm{c}}=$ (ácaros vivos test. - ácaros vivos trat.)/ácaros vivos test. $\mathrm{x} 100$.

${ }^{3} \mathrm{R}=$ Produção média de ovos/fêmeas. $\mathrm{R}=\mathrm{n}^{\underline{0}}$ ovos viáveis $/ \mathrm{n}^{\mathrm{o}}$ de fêmeas.

${ }^{4}$ Efeito na reprodução. $\mathrm{Er}=\mathrm{R}_{\text {Tratamento }} / \mathrm{R}_{\text {Testemunha }}$

${ }^{5}$ Efeito total ou adverso. $\mathrm{E}=100 \%-(100 \%$ - Mc) $\mathrm{x}$ Er.

${ }^{6}$ Classes toxicidade segundo a IOBC/WPRS: Classe $1=\mathrm{E}<30 \%$ (não nocivo); Classe $2=30<\mathrm{E}<80$ (levemente nocivo); Classe $3=80<\mathrm{E}<99$ (moderadamente nocivo); Classe 4 = E $>99 \%$ (nocivo). 
TABELA 6 - Número de ácaros Brevipalpus phoenicis (larvas, ninfas e adultos), em 25 folhas e 5 ramos de cafeeiro por parcela, aos 5 e 15 dias após a aplicação (DAA) e porcentagem de eficiência (\%E) dos tratamentos.

\begin{tabular}{|c|c|c|c|c|c|c|c|c|}
\hline \multirow{3}{*}{ Tratamentos $^{1}$} & \multicolumn{8}{|c|}{ Número de ácaros e porcentagem de eficiência dos tratamentos } \\
\hline & \multicolumn{4}{|c|}{ Folhas } & \multicolumn{4}{|c|}{ Ramos } \\
\hline & 5 DAA & $\% \mathbf{E}$ & 15 DAA & $\% \mathbf{E}$ & 5 DAA & $\% \mathbf{E}$ & 15 DAA & $\% \mathbf{E}$ \\
\hline 1 Testemunha & $18,5 \mathrm{a}^{2}$ & - & 14,8 a & - & $11,0 a^{2}$ & - & 10,8 a & - \\
\hline 2 Spirodiclofen & $2,3 \mathrm{c}$ & 87,8 & $0,0 \mathrm{~b}$ & 100,0 & 2,8 bc & 75,0 & $0,0 \quad \mathrm{c}$ & 100,0 \\
\hline 3 Azocyclotin PM & 0,0 с & 100,0 & $0,3 \mathrm{~b}$ & 98,3 & 2,3 bc & 79,5 & $4,3 \mathrm{ab}$ & 60,5 \\
\hline 4 Azocyclotin SC & $0,1 \quad \mathrm{c}$ & 94,6 & $0,8 \mathrm{~b}$ & 94,9 & $0,3 \quad \mathrm{c}$ & 97,7 & 3,0 bc & 72,1 \\
\hline 5 Fenbut. oxide & $2,3 \mathrm{c}$ & 87,8 & $0,3 \mathrm{~b}$ & 98,3 & $2,8 \mathrm{bc}$ & 75,0 & $4,0 \quad \mathrm{~b}$ & 62,8 \\
\hline 6 Hexythiazox & $11,5 \mathrm{~b}$ & 37,8 & 10,5 a & 28,8 & $4,5 \mathrm{ab}$ & 59,0 & $6,8 \mathrm{ab}$ & 37,2 \\
\hline CV(\%) & 28,7 & & 44,8 & & 41,9 & & 38,2 & \\
\hline
\end{tabular}

${ }^{1}$ Tratamentos: 1 - Testemunha; 2 - spirodiclofen (Envidor $240 \mathrm{SC}$ ) a $300 \mathrm{~mL} / \mathrm{ha} ; 3$ - azocyclotin (Peropal $250 \mathrm{PM}$ ) a $1000 \mathrm{~g} / \mathrm{ha} ; 4$ - azocyclotin (Caligur $500 \mathrm{SC}$ ) a $500 \mathrm{~mL} / \mathrm{ha} ; 5$ - fenbutatin oxide (Torque $500 \mathrm{SC}$ ) a $800 \mathrm{~mL} / \mathrm{ha} ; 6$ hexythiazox (Savey 500 PM) a $30 \mathrm{~g} / \mathrm{ha}$. Todos os produtos foram aplicados num volume de 1000 litros de calda/ha.

${ }^{2}$ Médias seguidas pela mesma letra na coluna, não diferem entre si, pelo teste de Duncan $(\mathrm{P}<0,05)$.

TABELA 7 - Número de ovos de Brevipalpus phoenicis, em 25 folhas e 5 ramos de cafeeiro por parcela, aos 5 e 15 dias após a aplicação (DAA) e porcentagem de eficiência (\%E) dos tratamentos.

\begin{tabular}{|c|c|c|c|c|c|c|c|c|}
\hline \multirow{3}{*}{ Tratamentos $^{1}$} & \multicolumn{8}{|c|}{ Número de ovos e porcentagem de eficiência dos tratamentos } \\
\hline & \multicolumn{4}{|c|}{ Folhas } & \multicolumn{4}{|c|}{ Ramos } \\
\hline & 5 DAA & $\% \mathrm{E}$ & 15 DAA & $\% \mathbf{E}$ & 5 DAA & $\% \mathbf{E}$ & 15 DAA & $\% \mathbf{E}$ \\
\hline 1 Testemunha & $22,0 a^{2}$ & - & $7,8 \mathrm{a}$ & - & $10,0 a^{2}$ & - & $27,0 \mathrm{a}$ & - \\
\hline 2 Spirodiclofen & $1,3 \mathrm{~b}$ & 94,3 & $0,0 \mathrm{~b}$ & 100,0 & 3,8 a & 62,5 & $0,5 \mathrm{c}$ & 98,1 \\
\hline 3 Azocyclotin PM & $4,3 \mathrm{~b}$ & 80,7 & $0,8 \mathrm{~b}$ & 90,3 & 6,3 a & 37,5 & 1,3 с & 95,4 \\
\hline 4 Azocyclotin SC & $4,0 \mathrm{~b}$ & 81,8 & $0,0 \mathrm{~b}$ & 100,0 & 4,5 a & 55,0 & $0,0 \mathrm{c}$ & 100,0 \\
\hline 5 Fenbut. Oxide & $7,0 \mathrm{~b}$ & 68,2 & $0,0 \mathrm{~b}$ & 100,0 & $4,8 \mathrm{a}$ & 52,5 & $1,0 \quad \mathrm{c}$ & 96,0 \\
\hline 6 Hexythiazox & $8,3 \mathrm{~b}$ & 62,5 & 4,8 a & 38,7 & $5,8 \mathrm{a}$ & 42,5 & $11,8 \mathrm{~b}$ & 56,0 \\
\hline CV(\%) & 42,2 & & 51,4 & & 35,6 & & 28,6 & \\
\hline
\end{tabular}

${ }^{1}$ Tratamentos: 1 - Testemunha; 2 - spirodiclofen (Envidor 240 SC) a $300 \mathrm{~mL} / \mathrm{ha} ; 3$ - azocyclotin (Peropal $250 \mathrm{PM}$ ) a 1000 g/ha; 4 - azocyclotin (Caligur 500 SC) a $500 \mathrm{~mL} / \mathrm{ha} ; 5$ - fenbutatin oxide (Torque 500 SC) a $800 \mathrm{~mL} / \mathrm{ha} ; 6$ hexythiazox (Savey 500 PM) a $30 \mathrm{~g} / \mathrm{ha}$. Todos os produtos foram aplicados num volume de 1000 litros de calda/ha.

${ }^{2}$ Médias seguidas pela mesma letra na coluna, não diferem entre si, pelo teste de Duncan $(P<0,05)$. 


\section{CONCLUSÕES}

a) Os acaricidas spirodiclofen (Envidor $240 \mathrm{SC}$ ) e azocyclotin (Peropal 250 PM e Caligur 500 SC) são eficientes como ovicidas no controle do ácaro da mancha-anular do cafeeiro, B. phoenicis;

b) $\mathrm{O}$ acaricida spirodiclofen (Envidor 240 SC) é eficiente ovicida no controle de $O$. ilicis;

c) Os acaricidas spirodiclofen (Envidor $240 \mathrm{SC}$ ) e azocyclotin (Peropal 250 PM e Caligur 500 SC) apresentam feito tópico e residual no controle das fases pós-embrionárias dos ácaros $B$. phoenicis e $O$. ilicis;

d) Em condições de campo, o spirodiclofen (Envidor 240 SC) e azocyclotin (Peropal 250 PM e Caligur 500 SC) são eficientes no controle de todas as fases pós-embrionárias do ácaro B. phoenicis;

e) O acaricida azocyclotin (Peropal $250 \mathrm{PM}$ e Caligur $500 \mathrm{SC}$ ) é nocivo aos ácaros predadores $E$. alatus, E. citrifolius e A. herbicolus;

f) Em laboratório, o spirodiclofen (Envidor 240 SC) apresenta seletividade fisiológica aos ácaros predadores E. alatus, E. citrifolius e A. herbicolus.

\section{AGRADECIMENTOS}

À Bayer CropScience pelo apoio financeiro e pela nossa participação no Club Elite BayCafé; ao Consórcio Brasileiro de Pesquisa e Desenvolvimento do Café - CBP\&D/Café e CNPq pelas bolsas concedidas.

\section{REFERÊNCIAS BIBLIOGRÁFICAS}

ABBOTT, W. S. A method of computing the effectiveness of an insecticide. Journal of Economic Entomology, Lanham, v. 18, p. 265-267, 1925.

AMARAL, J. F. O ácaro dos cafezais. Boletim da Superintendência dos Serviços do Café, São Paulo, v. 26, n. 296, p. 846-848, 1951.

CHAGAS, C. M. Associação do ácaro Brevipalpus phoenicis (Geijskes) à mancha anular do cafeeiro. $\mathbf{O}$ Biológico, São Paulo, v. 39, n. 9, p. 229-232, set. 1973.

CHIAVEGATO, L. G. Biologia do ácaro Brevipalpus phoenicis em citros. Pesquisa Agropecuária Brasileira, Brasília, v. 21, n. 8, p. 813-816, ago. 1986.
FIGUEIRA, A. R.; REIS, P. R.; CARVALHO, V. L.; PINTO, C. S. Coffee ringspot virus is becoming a real problem to brazilian coffee growers. In: INTERNATIONAL CONGRESS OF VIROLOGY, 10., 1996, Jerusalem, Israel. Abstracts... Jerusalem: [s.n.], 1996. p. 203.

HASSAN, S. A.; BIGLER, F.; BOGENSCHÜTZ, H.; BOLLER, E.; BRUN, J.; CALIS, J. N. M.; COREMANS-PELSENEER, J.; DUSO, C.; GROVE, A.; HEIMBACH, U.; HELYER, N.; HOKKANEN, H.; LEWIS, G. B.; MANSOUR, F.; MORETH, L.; POLGAR, L.; SAMSOE-PETERSEN, L.; SAUPHANOR, B.; STÄUBLI, A.; STERK, G.; VAINIO, A.; VEIRE, M. van de; VIGGIANI, G.; VOGT, H. Results of the sixth joint pesticide testing programme of the IOBC/WPRS: workig group "Pesticides and Beneficial Organisms". Entomophaga, Paris, v. 39, n. 1, p. 107-119, 1994.

INSTITUTO BRASILEIRO DO CAFÉ. Cultivo do café conilon. In: Cultura do café no Brasil: manual de recomendações. Rio de Janeiro: IBC/GERCA, 1985. p. 527-556, $580 \mathrm{p}$.

MATIELLO, J. B. Controle do ácaro vermelho do cafeeiro (Oligonychus $O$. ilicis) com novas formulações acaricidas. In: CONGRESSO BRASILEIRO DE PESQUISAS CAFEEIRAS, 26., 2000, Marília. Resumos... Rio de Janeiro: MA/PROCAFÉ, 2000. p. 26-27, 380 p.

MATIELLO, J. B. Novas condições de ocorrência de mancha anular do cafeeiro. In: CONGRESSO BRASILEIRO DE PESQUISAS CAFEEIRAS, 14., 1987, Campinas. Resumos... Rio de Janeiro: MIC/IBC, 1987. p. 6, 323 p.

McMURTRY, J. A.; CROFT, B. A. Life-styles of phytoseiid mites and their roles in biological control. Annual Review of Entomology, Palo Alto, v. 42, p. 291-321, 1997.

OLIVEIRA, C. A. L.; REIFF, E. T. Influência do volume de calda aplicada de acaricidas no controle do Brevipalpus phoenicis, transmissor da mancha anular do cafeeiro. In: CONGRESSO BRASILEIRO DE PESQUISAS CAFEEIRAS, 24., Poços de Caldas, 1998. Trabalhos Apresentados... Rio de Janeiro: MAA/SDR/PROCAFÉ/PNFC, 1998. p. 140, 319 p. 
OVERMEER, W. P. J.; ZON, A. Q. van. A standardized method for testing the side effects of pesticides on the predaceous mite, Amblyseius potentillae (Acarina: Phytoseiidae). Entomophaga, Paris, v. 27, n. 4, p. 357-64, 1982.

PALLINI FILHO, A.; MORAES, G. J.; BUENO, V. H. P. Ácaros associados ao cafeeiro (Coffea arabica L.) no Sul de Minas Gerais. Ciência e Prática, Lavras, v. 16, n. 3, p. 303-307, jul./set. 1992.

PAPA, G. Ocorrência, sintomas e controle do ácaro-daleprose, Brevipalpus phoenicis, (Geijskes, 1939) (Acari: Tenuipalpidae), na cultura do café. In: CONGRESSO BRASILEIRO DE PESQUISAS CAFEEIRAS, 23., Manhuaçu, 1997. Trabalhos Apresentados... Rio de Janeiro: MAA/SDR/PROCAFÉ/PNFC, 1997. p. 231233, 249 p.

REIS, P. R.; ALVES, E. B. Criação do ácaro predador Iphiseiodes zuluagai Denmark \& Muma (Acari: Phytoseiidae) em laboratório. Anais da Sociedade Entomológica do Brasil, Itabuna, v. 26, n. 3, p. 565568, dez. 1997.

REIS, P. R.; ALVES, E. B.; SOUSA, E. O. Biologia do ácaro-vermelho do cafeeiro Oligonychus ilicis (McGregor, 1917). Ciência e Agrotecnologia, Lavras, v. 21, n. 3, p. 260-266, jul./set. 1997.

REIS, P. R.; CHAGAS, S. J. R. Relação entre o ataque do ácaro-plano e da mancha-anular com indicadores da qualidade do café. Ciência e Agrotecnologia, Lavras, v. 25, n. 1, p. 72-76, jan./fev. 2001.

REIS, P. R.; CHIAVEGATO, L. G.; MORAES, G. J.; ALVES, E. B.; SOUSA, E. O. Seletividade de agroquímicos ao ácaro predador Iphiseiodes zuluagai Denmark \& Muma (Acari: Phytoseiidae). Anais da Sociedade Entomológica do Brasil, Itabuna, v. 27, n. 2, p. 265-274, jun. 1998.
REIS, P. R.; SOUZA, J. C. Pragas do cafeeiro. In: RENA, A. B.; MALAVOLTA, E.; ROCHA, M.; YAMADA, T. (Eds.). Cultura do cafeeiro: fatores que afetam a produtividade. Piracicaba: POTAFOS, 1986. p. 338-378, 447 p.

REIS, P. R.; SOUSA, E. O.; ALVES, E. B. Seletividade de produtos fitossanitários ao ácaro predador Euseius alatus DeLeon (Acari: Phytoseiidae). Revista Brasileira de Fruticultura, Jaboticabal, v. 21, n. 3, p. 350-355, dez. 1999.

REIS, P. R.; SOUZA, J. C.; PEDRO NETO, M.; TEODORO, A. V. Flutuação populacional do ácaro da mancha-anular do cafeeiro e seus inimigos naturais. In: SIMPÓSIO DE PESQUISA DOS CAFÉS DO BRASIL, 2000, Poços de Caldas. Resumos Expandidos... Brasília: EMBRAPA-CAFÉ, 2000a. v. 2, p. 1210-1212, 1490 p.

REIS, P. R.; SOUZA, J. C.; SOUSA, E. O.; TEODORO, A. V. Distribuição espacial do ácaro Brevipalpus phoenicis (Geijskes) (Acari: Tenuipalpidae) em cafeeiro (Coffea arabica L.). Anais da Sociedade Entomológica do Brasil, Itabuna, v. 29, n. 1, p. 177-183, mar. 2000b.

REIS, P. R.; SOUZA, J. C.; SOUSA, E. O.; TEODORO, A. V. Controle do Brevipalpus phoenicis em cafeeiro com produtos seletivos a ácaros predadores. Manejo Integrado de Plagas y Agroecologia, Costa Rica, v. 64, p. 55-61, jun. 2002.

WACHENDORFF, U.; NAUEN, R.; SCHNORBACH, H. J.; RAUCH, N.; ELBERT, A. The biological profile of spirodiclofen (Envidor): a new selective tetronic acid acaricide. Pflanzenschutz Nachrichten Bayer, Leverkusen, v. 55, n. 73, p. 149-176, 2002.

WOLF, C.; SCHNORBACH, H. J. Ecobiological profile of the acaricide spirodiclofen. Pflanzenschutz Nachrichten Bayer, Leverkusen, v. 55, n. 73, p. 177196, 2002. 